\title{
Associations between XRCC1 Gene Polymorphisms and Coronary Artery Disease: A Meta-Analysis
}

\author{
Wen-Qi Ma, Xi-Qiong Han, Xin Wang, Ying Wang, Yi Zhu, Nai-Feng Liu* \\ Department of Cardiology, Zhongda Hospital, School of Medicine, Southeast University, 87 Dingjiaqiao, \\ Nanjing, 210009, P.R. China \\ *liunf@seu.edu.cn
}

\section{Abstract}

Genetic variations that influence DNA repair efficiency may contribute to coronary artery disease (CAD) susceptibility. Previous studies have investigated whether there was evidence of an association between polymorphisms at the $X$-ray repair cross complementing 1 (XRCC1) gene and susceptibility to CAD, but findings have been inconclusive. We identified eligible studies through a comprehensive literature search to determine whether an association exists between $X R C C 1$ gene polymorphisms and CAD susceptibility. Findings were assessed using the odds ratio (OR) and corresponding $95 \%$ confidence interval

\section{OPEN ACCESS}

Citation: Ma W-Q, Han X-Q, Wang X, Wang Y, Zhu Y, Liu N-F (2016) Associations between XRCC1 Gene Polymorphisms and Coronary Artery Disease: A Meta-Analysis. PLoS ONE 11(11): e0166961. doi:10.1371/journal.pone.0166961

Editor: Robert M Lafrenie, Sudbury Regional Hospital, CANADA

Received: July 31, 2016

Accepted: November 6, 2016

Published: November 21, 2016

Copyright: @ 2016 Ma et al. This is an open access article distributed under the terms of the Creative Commons Attribution License, which permits unrestricted use, distribution, and reproduction in any medium, provided the original author and source are credited.

Data Availability Statement: All relevant data are within the paper and its Supporting Information files.

Funding: The authors received no specific funding for this work.

Competing Interests: The authors have declared that no competing interests exist.
$(\mathrm{Cl})$, which were calculated using a fixed- or random-effects model, based on the heterogeneity of the studies. Ten eligible studies were finally included in this meta-analysis. Our pooled analysis found that XRCC1 polymorphisms were significantly associated with CAD susceptibility under recessive (Arg194Trp: OR $=1.47,95 \% \mathrm{Cl}=1.13-1.93$; Arg399GIn: OR $=1.45,95 \% \mathrm{Cl}=1.12-1.89$ ), homozygous (Arg194Trp: OR $=1.37,95 \%$ $\mathrm{Cl}=1.03-1.81 ; \operatorname{Arg} 399 \mathrm{GIn}: \mathrm{OR}=1.56,95 \% \mathrm{Cl}=1.19-2.05$ ), and allele (Arg399GIn: $\mathrm{OR}=1.18,95 \% \mathrm{Cl}=1.06-1.32)$ genetic models. Following subgroup analysis by ethnicity, in Asian populations, we found evidence of associations between the XRCC1 Arg194Trp polymorphism and CAD under recessive and homozygous genetic models, and between the XRCC1 Arg399GIn polymorphism and CAD under recessive, homozygous, and allele genetic models. Subgroup analysis stratified by control source revealed associations between the Arg194Trp and Arg399GIn polymorphisms and susceptibility to CAD under recessive and homozygous modes of inheritance, respectively. In addition, subgroup analysis stratified by sample size found that findings of the Arg194Trp polymorphism in large sample sizes were comparable to those found using pooled eligible studies. Based on our meta-analysis, we concluded that the XRCC1 gene polymorphisms, Arg194Trp and Arg399GIn, are associated with CAD susceptibility, specifically in Asian populations. However, additional, comprehensive and well-designed studies are warranted to confirm these findings. 


\section{Introduction}

Coronary artery disease (CAD), which is recognized as a major public health problem, has high mortality and morbidity worldwide [1]. The main underlying cause of CAD is atherosclerosis. As a chronic, progressive, and multifactorial disease, a variety of complex mechanisms affects the initiation and progression of atherosclerosis and plaque vulnerability [2, 3]. One possible mechanism for the progression of atherosclerosis is via DNA damage, which is caused by the generation of oxidative stress, the accumulation of reactive oxygen species (ROS), the metabolism of toxic byproducts, and ionizing radiation [4-6]. Genome integrity is probably influenced by endogenous and exogenous DNA damage, and sustained deleterious effects that block both replication and transcription of DNA may lead to mutations and chromosomal aberrations [7, 8]. However, several DNA damage response pathways, such as base excision repair (BER), nucleotide excision repair (NER), and single strand break repair (SSBR), fix such DNA damage [9]. One of the most important components for the efficient repair of SSBR and BER is X-ray repair cross complementing protein 1 (XRCC1) [10-12]. It has a role as a scaffold coordinating other proteins in the DNA repair complex. The XRCC1 gene is located on chromosome 19q13.2-13.3, it consists of 17 exons and encodes a protein of 633 amino acids [13].

Two XRCC1 gene polymorphisms, Arg194Trp (rs1799782) and Arg399Gln (rs25487), which are located at codon 194 of exon 6 and at codon 399 of exon 10 respectively, are reported to affect the expression of $X R C C 1$ and subsequently influence the DNA repair capacity $[14,15]$. Furthermore, previous studies have found that individuals with DNA repair deficiencies show an increased sensitivity to CAD [16-18]. In addition, several epidemiological studies have revealed that these two variations in the XRCC1 gene influence DNA repair and are associated with an increased susceptibility to CAD [19-21]. For example, Bazo et al. [22] demonstrated that individuals possessing the T allele in Arg194Trp or the A allele in Arg399Gln have an increased likelihood of CAD compared to that found in individuals with the wild-type alleles of either polymorphisms. However, other studies did not consistently find evidence of an association between polymorphisms in the XRCC1 gene and the likelihood of developing CAD $[18,23]$.

Whether a correlation exists between variants in the DNA repair gene XRCC1 and susceptibility to CAD remains controversial and inconclusive. Furthermore, no relevant meta-analysis or genome-wide association studies (GWAS) have been published on this subject. Consequently, to derive a more comprehensive estimation of the role of XRCC1 gene polymorphisms in CAD susceptibility, we identified all eligible studies and performed this meta-analysis.

\section{Methods and Materials Literature search strategy}

The literature search was performed by two authors (Ma and Zhu). Comprehensive electronic databases including PubMed, EMBASE, Web of Science, ScienceDirect, Cochrane Library, and Ovid were systematically searched for eligible studies up to July 31,2016. Our search strategy was based on finding keywords or topic-based classifications in the title and abstract of published studies. Keywords and terms used were ("XRCC1" OR "X-ray repair cross-complementing group 1" OR “Arg194Trp" OR "rs1799782" OR “Arg399Gln" OR "rs25487”) and ("genetic polymorphism" OR "allele" OR "genotype" OR "variant") and ("coronary artery disease" OR "myocardial infarction" OR "angina" OR "atherosclerosis"). References in eligible studies were also examined as a secondary source to identify eligible studies. Manual searching of relevant journals and screening the reference lists were performed by one author (Liu). 


\section{Inclusion and exclusion criteria}

Studies included in our meta-analysis were selected according to the following inclusion criteria: (1) the study examined the correlation between the XRCC1 Arg194Trp and Arg399Gln polymorphisms and CAD; (2) patients with CAD were documented using angiographic evidence of at least $50 \%$ stenosis of one major coronary artery, myocardial infarction, angina, or a history of coronary artery bypass surgery; (3) the total number of cases and controls, distribution of genotypes, or other relevant data could be extracted from the references; and (4) the publication language was in English. Studies were excluded if they met any of the following criteria: (1) publications were abstracts, letters to the editor, animal studies, or reviews; (2) the study presented data that overlapped with a previous publication; and (3) the study presented either uninformative data or did not provide genotype frequencies.

\section{Data extraction}

The data from eligible studies were extracted by two independent authors (Ma and Han). Disagreements regarding study selection were resolved by a third author (Liu). Critical data that could not be derived from the references were obtained by directly contacting the authors. We collected the following information: author, year of publication, ethnicity, country, the number of cases and controls, baseline characteristics of the patients, genotyping methods, genotype frequencies in cases and controls, the origin of the controls, and measurements of HardyWeinberg equilibrium (HWE) from controls.

\section{Quality assessment}

The quality of eligible studies was assessed by one author (Han), according to the NewcastleOttawa scale (NOS) for genetic association studies [24]. NOS quality scores ranged between 0 and 9 stars. Studies with a score $\geq 7$ were considered high quality, while studies with a score $\leq 5$ were deemed low quality.

\section{Statistical analysis}

All statistical analyses were performed using Review Manager v5.2 (The Cochrane Collaboration, Oxford, UK) and Stata 12.0 (Stata Corporation, College Station, Texas, USA). Pooled odds ratios (ORs) and 95\% confidence intervals (CIs) were calculated to evaluate the robustness of the association between XRCC1 gene polymorphisms and CAD susceptibility. Tests under dominant (Arg194Trp: CT + TT vs. CC; Arg399Gln: GA + AA vs. GG), recessive (Arg194Trp: TT vs. CT + CC; Arg399Gln: AA vs. GA + GG), homozygous (Arg194Trp: TT vs. CC; Arg399Gln: AA vs. GG), heterozygous (Arg194Trp: CT vs. CC; Arg399Gln: GA vs. GG), and allele (Arg194Trp: T vs. C; Arg399Gln: A vs. G) genetic models were performed and ORs were determined. The Cochrane Q-test and index $\left(\mathrm{I}^{2}\right)$ were used to examine heterogeneity among studies with a $P>0.10$ in the Q-test indicating no heterogeneity. $\mathrm{I}^{2}$ was used to estimate the total variation across studies where an $\mathrm{I}^{2}<25 \%$ was considered to have a low level of heterogeneity, an $\mathrm{I}^{2}$ between $25 \%$ and $50 \%$ indicated moderate heterogeneity, and an $\mathrm{I}^{2}>50 \%$ represented a high-level of heterogeneity. A fixed- or random-effects model was performed depending on the observed heterogeneity of the studies. Subgroup analyses were performed to identify potential sources of heterogeneity. Sensitivity analysis was performed to assess the stability of individual studies, and publication bias was determined with funnel plots and Egger's linear regression test. 


\section{Results}

\section{Selection and characteristics of studies}

Fifty-nine articles were selected following an initial search, of which 44 articles were excluded because of duplicate records. Ten articles remained from the original literature search after screening the titles and abstracts. Of these studies, one article was excluded because it was a review and two other articles were excluded because of insufficient data. Finally, seven articles that contained ten studies were retained [18-23, 25]. The flow diagram of our literature selection process is shown in Fig 1.

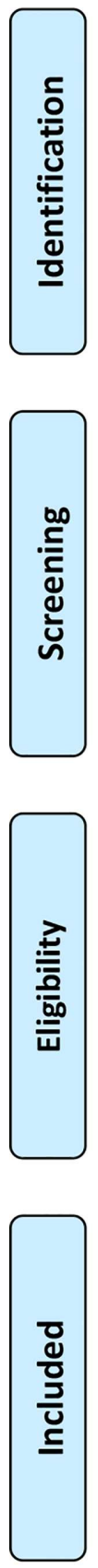



$(n=59)$
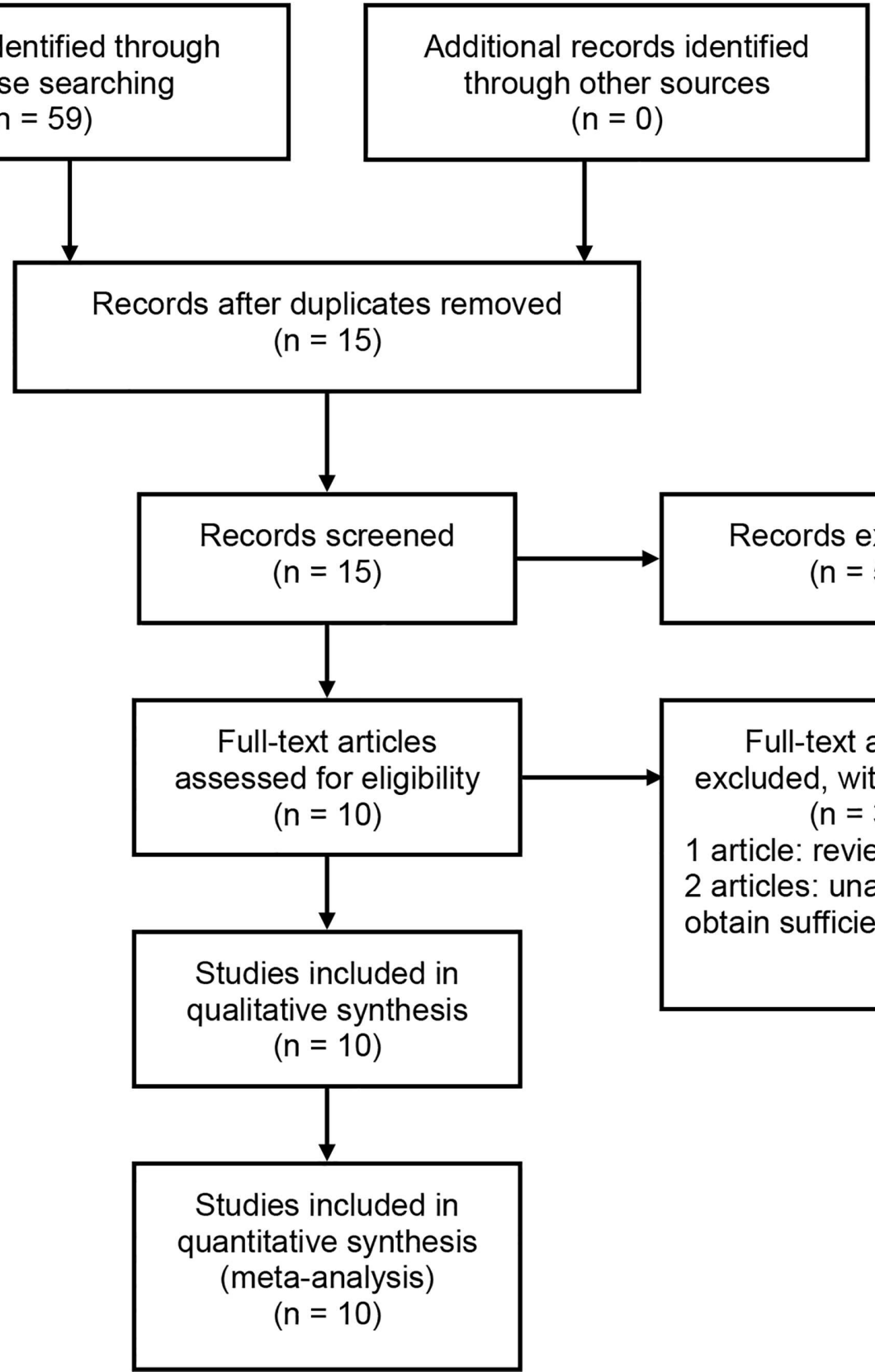

Records excluded $(n=5)$

Fig 1. Flow chart to identify eligible studies for meta-analysis.

doi:10.1371/journal.pone.0166961.g001 
Information of the selected studies is shown in Table 1. For the Arg194Trp polymorphism, 1,490 cases and 1,458 controls were available from four studies, whereas for the Arg399Gln polymorphism, 1,862 cases and 1,568 controls were available from six studies. The countries of eligible studies included Brazil, Pakistan, China, Turkey, India, and Iran. Two studies used Caucasian samples and six studies used Asian samples. The controls of six studies were hospital-based, whereas those of the other four studies were population-based. Control cohorts from all studies were in HWE $(P>0.05)$.

Table 1. Characteristics of eligible studies included in the meta-analysis.

\begin{tabular}{|c|c|c|c|c|c|c|c|c|c|c|c|}
\hline $\begin{array}{c}\text { First Author/ } \\
\text { Year }\end{array}$ & Country & Ethnicity & $\begin{array}{c}\text { Disease } \\
\text { Type }\end{array}$ & $\begin{array}{l}\text { Clinical Criteria } \\
\text { for Patients }\end{array}$ & $\begin{array}{l}\text { Control } \\
\text { Source }\end{array}$ & $\begin{array}{l}\text { Genotyping } \\
\text { Methods }\end{array}$ & $\begin{array}{l}\text { Sample } \\
\text { Size }\end{array}$ & $\begin{array}{l}\text { No. of } \\
\text { Cases }\end{array}$ & $\begin{array}{l}\text { No. of } \\
\text { Controls }\end{array}$ & $\begin{array}{c}P_{H W E} \\
Y / N\end{array}$ & NOS \\
\hline Arg194Trp & & & & & & & & $\begin{array}{c}\text { CC/CT/ } \\
\text { TT }\end{array}$ & CC/CT/TT & & \\
\hline Bazo 2011[22] & Brazil & Mixed & CAD & $\begin{array}{l}\text { stenosis }>50 \% \text { in } \\
\text { at least one } \\
\text { coronary artery }\end{array}$ & $\mathrm{HB}$ & PCR & $46 / 39$ & $40 / 6 / 0$ & $28 / 10 / 1$ & $\mathrm{Y}$ & 6 \\
\hline Yu 2014[20] & China & Asian & CAD & $\begin{array}{c}\text { stenosis }>50 \% \text { in } \\
\text { at least one major } \\
\text { coronary artery or } \\
\text { major branches }\end{array}$ & $\mathrm{HB}$ & PCR-LDR & $\begin{array}{l}1142 / \\
1106\end{array}$ & $\begin{array}{l}517 / \\
486 / \\
139\end{array}$ & $\begin{array}{c}483 / 531 / \\
92\end{array}$ & $\mathrm{Y}$ & 6 \\
\hline $\begin{array}{l}\text { Pahlavanneshn } \\
\text { 2016[19] }\end{array}$ & Iran & Asian & CAD & $\begin{array}{l}\text { stenosis }>50 \% \text { in } \\
\text { at least one } \\
\text { coronary artery }\end{array}$ & $\mathrm{HB}$ & PCR-RFLP & $203 / 207$ & $\begin{array}{c}155 / 44 / \\
4\end{array}$ & $185 / 19 / 3$ & $\mathrm{Y}$ & 6 \\
\hline $\begin{array}{c}\text { Hameed } 2016 \\
{[25]}\end{array}$ & Pakistan & Asian & $\begin{array}{c}\text { AMI, stable } \\
\text { and } \\
\text { unstable } \\
\text { angina }\end{array}$ & $\begin{array}{l}\text { stenosis }>50 \% \text { in } \\
\text { at least one } \\
\text { coronary artery }\end{array}$ & PB & PCR-RFLP & $99 / 106$ & $94 / 3 / 2$ & $99 / 4 / 3$ & $\mathrm{Y}$ & 7 \\
\hline Arg399GIn & & & & & & & & $\begin{array}{c}\text { AA/ } \\
\text { AG/GG }\end{array}$ & $\begin{array}{c}\text { AA/AG/ } \\
\text { GG }\end{array}$ & & \\
\hline Guven $2007^{[23]}$ & Turkey & Caucasian & CAD & $\begin{array}{l}\text { stenosis }>50 \% \text { in } \\
\text { at least one } \\
\text { coronary artery }\end{array}$ & PB & PCR-RFLP & $147 / 48$ & $\begin{array}{c}50 / 76 / \\
21\end{array}$ & $12 / 33 / 3$ & $\mathrm{Y}$ & 6 \\
\hline Bazo 2011[22] & Brazil & mixed & CAD & $\begin{array}{l}\text { stenosis }>50 \% \text { in } \\
\text { at least one } \\
\text { coronary artery }\end{array}$ & $\mathrm{HB}$ & PCR & $117 / 52$ & $\begin{array}{c}25 / 76 / \\
16\end{array}$ & $20 / 28 / 4$ & $\mathrm{Y}$ & 6 \\
\hline Narne 2013[21] & India & Asian & $\mathrm{CAD}, \mathrm{MI}$ & $\begin{array}{l}\text { Diabetic patients } \\
\text { with stenosis } \\
>50 \% \text { in at least } \\
\text { one coronary } \\
\text { artery }\end{array}$ & $\mathrm{HB}$ & PCR & $160 / 121$ & $\begin{array}{l}52 / 84 / \\
24\end{array}$ & $58 / 49 / 14$ & $\mathrm{Y}$ & 6 \\
\hline $\begin{array}{c}\text { Gokkusu } 2013 \\
{[18]}\end{array}$ & Turkey & Caucasian & CAD, ACS & $\begin{array}{c}\text { stenosis }>50 \% \text { in } \\
\text { one major } \\
\text { coronary artery }\end{array}$ & PB & PCR & $197 / 135$ & $\begin{array}{c}169 / 27 / \\
1\end{array}$ & $119 / 16 / 0$ & $Y$ & 7 \\
\hline Yu 2014[20] & China & Asian & CAD & $\begin{array}{l}\text { stenosis }>50 \% \text { in } \\
\text { one major } \\
\text { coronary artery or } \\
\text { major branches }\end{array}$ & $\mathrm{HB}$ & PCR-LDR & $\begin{array}{l}1142 / \\
1106\end{array}$ & $\begin{array}{c}625 / \\
437 / 80\end{array}$ & $\begin{array}{c}627 / 419 / \\
60\end{array}$ & $\mathrm{Y}$ & 6 \\
\hline $\begin{array}{c}\text { Hameed } 2016 \\
\text { [25] }\end{array}$ & Pakistan & Asian & $\begin{array}{c}\text { AMI, stable } \\
\text { and } \\
\text { unstable } \\
\text { angina }\end{array}$ & $\begin{array}{l}\text { stenosis }>50 \% \text { in } \\
\text { at least one } \\
\text { coronary artery }\end{array}$ & PB & PCR-RFLP & 99/106 & $\begin{array}{c}40 / 28 / \\
31\end{array}$ & $53 / 30 / 23$ & $\mathrm{Y}$ & 7 \\
\hline
\end{tabular}

Abbreviations: CAD, coronary artery disease; ACS, acute coronary artery syndrome; AMI, acute myocardial infarction; MI, myocardial infarction; PCR, polymerase chain reaction; PCR-RFLP, polymerase chain reaction-restriction fragment length polymorphism; PCR-LDR, polymerase chain reaction-ligase detection reaction; HWE, Hardy-Weinberg equilibrium; PB, population-based; HB, hospital-based; NOS, Newcastle-Ottawa quality scale.

doi:10.1371/journal.pone.0166961.t001 


\section{Meta-analysis results}

Associations between XRCC1 gene polymorphisms and CAD susceptibility are shown in Table 2. Subgroup analyses were performed based on the source of the controls (hospitalbased and population-based controls), ethnicity (Asian and non-Asian populations), and sample size (studies with $\geq 300$ subjects were categorized as "large" and studies with $<300$ subjects were categorized as "small") to detect sources of heterogeneity (Table 2).

Table 2. Summary of meta-analysis of the association between XRCC1 gene polymorphisms and coronary artery disease.

\begin{tabular}{|c|c|c|c|c|c|c|c|c|c|}
\hline \multirow[t]{2}{*}{ Genetic Model } & \multirow[t]{2}{*}{ Overall and Subgroup } & \multirow[b]{2}{*}{$\mathbf{N}$} & \multicolumn{3}{|c|}{ Arg194Trp } & \multirow[b]{2}{*}{$\mathbf{N}$} & \multicolumn{3}{|c|}{ Arg399GIn } \\
\hline & & & OR $(95 \% \mathrm{Cl})$ & $I^{2}(\%)$ & $\boldsymbol{P}$ & & OR $(95 \% \mathrm{Cl})$ & $I^{2}(\%)$ & $\boldsymbol{P}$ \\
\hline & Overall & 4 & $1.02(0.51-2.07)$ & $81 \%$ & 0.001 & 6 & $1.32(0.97-1.79)$ & $56 \%$ & 0.040 \\
\hline & PB & 1 & $0.75(0.23-2.45)$ & $\mathrm{N} / \mathrm{A}$ & $\mathrm{N} / \mathrm{A}$ & 3 & $1.11(0.67-1.77)$ & $37 \%$ & 0.210 \\
\hline & HB & 3 & $1.08(0.46-2.52)$ & $87 \%$ & 0.000 & 3 & $1.56(0.94-2.59)$ & $76 \%$ & 0.020 \\
\hline \multirow[t]{7}{*}{ Dominant model } & Large sample size & 2 & $0.99(0.56-4.10)$ & $92 \%$ & 0.000 & 2 & $1.09(0.93-1.28)$ & $0 \%$ & 0.710 \\
\hline & Small sample size & 2 & $1.28(0.23-1.17)$ & $0 \%$ & 0.410 & 4 & $1.47(0.91-2.38)$ & $59 \%$ & 0.060 \\
\hline & Asian populations & 3 & $1.18(0.59-2.82)$ & $84 \%$ & 0.002 & 3 & $1.37(0.94-1.98)$ & $63 \%$ & 0.070 \\
\hline & non-Asian populations & 1 & $0.38(0.13-1.15)$ & $\mathrm{N} / \mathrm{A}$ & $\mathrm{N} / \mathrm{A}$ & 3 & $1.23(0.61-2.47)$ & $66 \%$ & 0.050 \\
\hline & Overall & 4 & $1.47(1.13-1.93)$ & $0 \%$ & 0.630 & 6 & $1.45(1.12-1.89)$ & $0 \%$ & 0.910 \\
\hline & PB & 1 & $0.71(0.12-4.33)$ & $\mathrm{N} / \mathrm{A}$ & $\mathrm{N} / \mathrm{A}$ & 3 & $2.09(1.05-3.16)$ & $0 \%$ & 0.840 \\
\hline & HB & 3 & $1.50(1.14-1.97)$ & $0 \%$ & 0.580 & 3 & $1.36(1.01-1.83)$ & $0 \%$ & 0.830 \\
\hline \multirow[t]{7}{*}{ Recessive model } & Large sample size & 2 & $1.34(1.16-2.00)$ & $0 \%$ & 0.890 & 2 & $1.32(0.94-1.86)$ & $0 \%$ & 0.780 \\
\hline & Small sample size & 2 & $0.55(0.12-2.62)$ & $0 \%$ & 0.620 & 4 & $1.66(1.10-2.49)$ & $0 \%$ & 0.850 \\
\hline & Asian populations & 3 & $1.32(1.14-1.96)$ & $0 \%$ & 0.710 & 3 & $1.38(1.04-1.82)$ & $0 \%$ & 0.830 \\
\hline & non-Asian populations & 1 & $0.28(0.01-6.97)$ & $\mathrm{N} / \mathrm{A}$ & $N / A$ & 3 & $2.16(0.95-4.90)$ & $0 \%$ & 0.950 \\
\hline & Overall & 4 & $1.03(0.47-2.27)$ & $82 \%$ & 0.000 & 6 & $1.23(0.89-1.70)$ & $56 \%$ & 0.040 \\
\hline & PB & 1 & $1.13(0.17-3.62)$ & $\mathrm{N} / \mathrm{A}$ & $\mathrm{N} / \mathrm{A}$ & 3 & $0.96(0.59-1.57)$ & $34 \%$ & 0.220 \\
\hline & HB & 3 & $1.08(0.43-2.70)$ & $88 \%$ & 0.000 & 3 & $1.51(0.90-2.54)$ & $74 \%$ & 0.020 \\
\hline \multirow[t]{7}{*}{ Heterozygous model } & Large sample size & 2 & $1.49(0.47-4.69)$ & $93 \%$ & 0.000 & 2 & $1.05(0.89-1.25)$ & $0 \%$ & 0.720 \\
\hline & Small sample size & 2 & $1.27(0.21-1.29)$ & $0 \%$ & 0.510 & 4 & $1.33(0.76-2.34)$ & $66 \%$ & 0.030 \\
\hline & Asian populations & 3 & $1.30(0.51-3.31)$ & $86 \%$ & 0.000 & 3 & $1.28(0.87-1.89)$ & $59 \%$ & 0.090 \\
\hline & non-Asian populations & 1 & $0.42(0.14-1.92)$ & $\mathrm{N} / \mathrm{A}$ & $\mathrm{N} / \mathrm{A}$ & 3 & $1.13(0.54-2.39)$ & $70 \%$ & 0.040 \\
\hline & Overall & 4 & $1.37(1.03-1.81)$ & $0 \%$ & 0.630 & 6 & $1.56(1.19-2.05)$ & $0 \%$ & 0.780 \\
\hline & PB & 1 & $0.70(0.11-4.30)$ & $\mathrm{N} / \mathrm{A}$ & $\mathrm{N} / \mathrm{A}$ & 3 & $1.78(0.98-3.23)$ & $0 \%$ & 0.993 \\
\hline & HB & 3 & $1.39(1.05-1.85)$ & $0 \%$ & 0.550 & 3 & $1.51(1.11-2.05)$ & $10 \%$ & 0.330 \\
\hline \multirow[t]{7}{*}{ Homozygous model } & Large sample size & 2 & $1.42(1.07-1.89)$ & $0 \%$ & 0.880 & 2 & $1.35(0.95-1.91)$ & $0 \%$ & 0.780 \\
\hline & Small sample size & 2 & $0.53(0.11-2.49)$ & $0 \%$ & 0.560 & 4 & $1.97(1.26-3.06)$ & $0 \%$ & 0.870 \\
\hline & Asian populations & 3 & $1.39(1.05-1.85)$ & $0 \%$ & 0.750 & 3 & $1.49(1.11-1.98)$ & $0 \%$ & 0.590 \\
\hline & non-Asian populations & 1 & $0.23(0.01-5.97)$ & $\mathrm{N} / \mathrm{A}$ & $\mathrm{N} / \mathrm{A}$ & 3 & $2.37(0.98-5.74)$ & $0 \%$ & 0.790 \\
\hline & Overall & 4 & $1.04(0.58-1.86)$ & $77 \%$ & 0.004 & 6 & $1.18(1.06-1.32)$ & $24 \%$ & 0.250 \\
\hline & PB & 1 & $1.15(0.28-1.98)$ & $\mathrm{N} / \mathrm{A}$ & $\mathrm{N} / \mathrm{A}$ & 3 & $1.25(0.95-1.65)$ & $0 \%$ & 0.410 \\
\hline & HB & 3 & $1.11(0.55-2.23)$ & $84 \%$ & 0.002 & 3 & $1.17(1.04-1.32)$ & $56 \%$ & 0.100 \\
\hline \multirow[t]{4}{*}{ Allele model } & Large sample size & 2 & $1.04(0.70-3.18)$ & $89 \%$ & 0.003 & 2 & $1.11(0.97-1.26)$ & $0 \%$ & 0.670 \\
\hline & Small sample size & 2 & $1.24(0.27-1.10)$ & $0 \%$ & 0.370 & 4 & $1.40(1.14-1.72)$ & $0 \%$ & 0.420 \\
\hline & Asian populations & 3 & $1.28(0.17-2.33)$ & $79 \%$ & 0.009 & 3 & $1.17(1.04-1.32)$ & $53 \%$ & 0.120 \\
\hline & non-Asian populations & 1 & $0.38(0.14-1.08)$ & $\mathrm{N} / \mathrm{A}$ & N/A & 3 & $1.26(0.94-1.69)$ & $7 \%$ & 0.340 \\
\hline
\end{tabular}

Abbreviations: XRCC1, X-ray repair cross complementing protein 1; N, number of studies; OR, odds ratio; $\mathrm{Cl}$, confidence interval; $P, P$ value for association; PB, Population-based; HB, Hospital-based; N/A, not available.

doi:10.1371/journal.pone.0166961.t002 
A

\begin{tabular}{lrrrrr} 
& \multicolumn{4}{c}{ case } & \multicolumn{3}{c}{ Control } \\
Study or Subgroup & Events & Total & Events & Total & Weight \\
\hline Bazo AP 2011 & 6 & 46 & 11 & 39 & $18.8 \%$ \\
Yu 2014 & 625 & 1142 & 623 & 1106 & $34.5 \%$ \\
Pahlavanneshan T 2015 & 48 & 203 & 22 & 207 & $29.0 \%$ \\
Hameed H 2016 & 5 & 99 & 7 & 106 & $17.7 \%$ \\
& & & & & \\
Total (95\% Cl) & & 1490 & & 1458 & $100.0 \%$ \\
Total events & 684 & 663 & \\
Heterogeneity: Tau $^{2}=0.37 ;$ Chi $^{2}=15.44$, df $=3(P=0.001) ;\left.\right|^{2}=81 \%$ \\
Test for overall effect: $Z=0.07(P=0.95)$
\end{tabular}

Odds Ratio

M-H, Random, $95 \%$ Cl Year

$0.38[0.13,1.15] 2011$

$0.94[0.79,1.11] 2014$

$2.60[1.51,4.50] 2015$

$0.75[0.23,2.45] 2016$

$1.02[0.51,2.07]$

Heterogeneity: $\mathrm{Tau}^{2}=0.37 ; \mathrm{Chi}^{2}=15.44, \mathrm{df}=3(\mathrm{P}=0.001) ; \mathrm{I}^{2}=81 \%$

Test for overall effect: $Z=0.07(P=0.95)$

B

Study or Subgroup

Case

Control

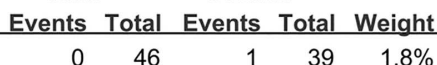

Bazo AP 2011

Yu 2014

Pahlavanneshan T 2015

Hameed H 2016

1391142

$4 \quad 203$

$92 \quad 1106 \quad 91.8 \%$

$\begin{array}{rrr}9 & 1106 & 91.8 \% \\ 3 & 207 & 3.3 \%\end{array}$

299

$3 \quad 106 \quad 3.2 \%$

Total $(95 \% \mathrm{Cl})$

1490

$1458 \quad 100.0 \%$

Total events

145

99

Heterogeneity: $\mathrm{Chi}^{2}=1.74, \mathrm{df}=3(\mathrm{P}=0.63) ; \mathrm{I}^{2}=0 \%$

Test for overall effect: $Z=2.84(P=0.005)$

Odds Ratio

M-H, Fixed, 95\% Cl Year

$0.28[0.01,6.97] 2011$

$1.53[1.16,2.02] 2014$

$1.37[0.30,6.19] 2015$

$0.71[0.12,4.33] 2016$

$1.47[1.13,1.93]$
Odds Ratio M-H, Random, $95 \% \mathrm{Cl}$

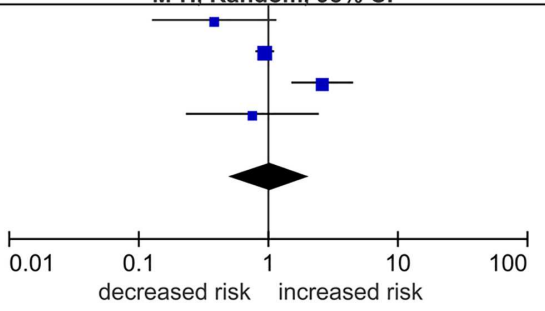

C

Study or Subgroup

Case

Control

Odds Ratio

Events Total Events Total Weight M-H, Random, 95\% Cl Year

Bazo AP 2011

Yu 2014

Pahlavanneshan T 2015

$\begin{array}{lllll}6 & 46 & 10 & 38 & 20.5 \%\end{array}$

$\begin{array}{lllll}486 & 1003 & 531 & 1014 & 34.7 \%\end{array}$

$0.42[0.14,1.29] 2011$

$0.86[0.72,1.02] 2014$

$2.76[1.55,4.93] 2015$

Hameed H 2016

$\begin{array}{rrrrr}44 & 199 & 19 & 204 & 29.6 \% \\ 3 & 97 & 4 & 103 & 15.2 \%\end{array}$

$0.79[0.17,3.62] 2016$

Total $(95 \% \mathrm{Cl})$

1345

$1359100.0 \%$

$1.03[0.47,2.27]$

Heterogeneity: $\mathrm{Tau}^{2}=0.46 ; \mathrm{Chi}^{2}=16.47, \mathrm{df}=3(\mathrm{P}=0.0009) ; \mathrm{I}^{2}=82 \%$

Test for overall effect: $Z=0.08(P=0.93)$



D

Case

Control

Odds Ratio

Odds Ratio M-H, Random, 95\% Cl

Study or Subgroup

Events Total Events Total Weight

Bazo AP 2011

Yu 2014

Pahlavanneshan T 2015

Hameed H 2016

040

$129 \quad 2.0 \%$

$\begin{array}{lllll}139 & 656 & 92 & 575 & 91.4 \%\end{array}$

$4 \quad 159$

$\begin{array}{rrr}92 & 575 & 91.4 \% \\ 3 & 188 & 3.2 \%\end{array}$

296

310

$3.4 \%$

Total $(95 \% \mathrm{Cl})$

951

Total events

145

(a)

Test for overall effect: $Z=2.20(P=0.03)$

M-H, Fixed, 95\% Cl Year $0.23[0.01,5.97] 2011$ $1.41[1.05,1.89] 2014$ $1.59[0.35,7.22] 2015$

$0.70[0.11,4.30] 2016$

$1.37[1.03,1.81]$

$894 \quad 100.0 \%$

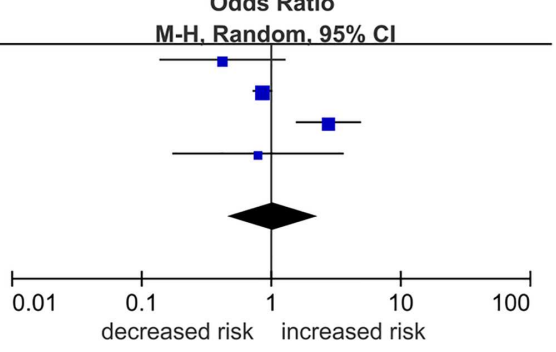

E

Study or Subgroup

Case Control

Odds Ratio Events Total Events Total Weight M-H, Random, 95\% Cl Year

Bazo AP 2011

Yu 2014

$\begin{array}{lllll}6 & 92 & 12 & 78 & 17.0 \%\end{array}$

$\begin{array}{lllll}764 & 2284 & 715 & 2212 & 36.2 \%\end{array}$

Pahlavanneshan T 2015

$\begin{array}{lllll}52 & 406 & 25 & 414 & 28.9 \%\end{array}$

Hameed H 2016

$\begin{array}{lllll}7 & 198 & 10 & 212 & 17.9 \%\end{array}$

Total $(95 \% \mathrm{Cl})$

2980

Heterogeneity: $\mathrm{Tau}^{2}=0.24 ; \mathrm{Chi}^{2}=13.30, \mathrm{df}=3(\mathrm{P}=0.004) ; \mathrm{I}^{2}=77 \%$

Test for overall effect: $Z=0.14(P=0.89)$

$0.38[0.14,1.08] 2011$

$1.05[0.93,1.19] 2014$

$2.29[1.39,3.76] 2015$

$0.74[0.28,1.98] 2016$

$1.04[0.58,1.86]$

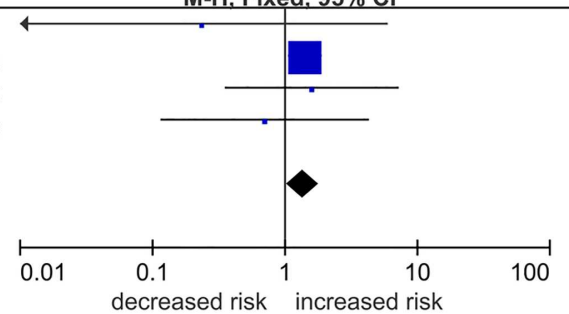

$2916 \quad 100.0 \%$

decreased risk increased risk

Odds Ratio $\mathrm{M}-\mathrm{H}$, Random, $95 \% \mathrm{Cl}$

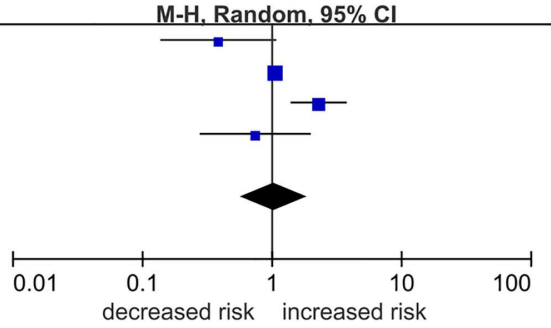


Fig 2. Forest plots for XRCC1 Arg194Trp polymorphism and coronary artery disease susceptibility in the genetic models: (A) Dominant (CT + TT vs. CC), (B) Recessive (TT vs. CT + CC), (C) Heterozygous (TC vs. CC), (D) Homozygous (TT vs. CC), and (E) Allele (T allele vs. $\mathrm{C}$ allele). Abbreviations: XRCC1, X-ray repair cross complementing protein 1; $\mathrm{Cl}$, confidence interval.

doi:10.1371/journal.pone.0166961.g002

For the XRCC1 Arg194Trp polymorphism, we found an increased likelihood of CAD susceptibility in recessive (TT vs. CT + CC: $\mathrm{OR}=1.47,95 \% \mathrm{CI}=1.13-1.93$ ) and homozygous (TT vs. $\mathrm{CC}: \mathrm{OR}=1.37,95 \% \mathrm{CI}=1.03-1.81)$ genetic models when cohorts from all eligible studies were pooled (Table 2; Fig 2). We found no evidence of an association between CAD and dominant (CT + TT vs. CC: $\mathrm{OR}=1.02,95 \% \mathrm{CI}=0.51-2.07)$, heterozygous (CT vs. $\mathrm{CC}: \mathrm{OR}=1.03$, $95 \% \mathrm{CI}=0.47-2.27$ ), or allele ( $\mathrm{T}$ vs. $\mathrm{C}: \mathrm{OR}=1.04,95 \% \mathrm{CI}=0.58-1.86$ ) genetic models. Similarly, pooled analysis found evidence of association between the XRCC1 Arg399Gln polymorphism and CAD susceptibility in recessive (AA vs. $\mathrm{GA}+\mathrm{GG}$ : $\mathrm{OR}=1.45,95 \% \mathrm{CI}=1.12-1.89$ ), homozygous (AA vs. GG: $\mathrm{OR}=1.56,95 \% \mathrm{CI}=1.19-2.05$ ), and allele (A vs. $\mathrm{G}$ : $\mathrm{OR}=1.18,95 \%$ $\mathrm{CI}=1.06-1.32)$ genetic models, but no evidence of association was identified under dominant and heterozygous models of inheritance (Table 2; Fig 3). We found no between-study heterogeneity under recessive (Arg194Trp: TT vs. CT $+\mathrm{CC}, \mathrm{I}^{2}=0 \%, P_{\text {Heterogeneity }}=0.630$; Arg399Gln: AA vs. GA + GG, $\mathrm{I}^{2}=0 \%, P_{\text {Heterogeneity }}=0.910$ ), homozygous (Arg194Trp: TT vs. CC, $\mathrm{I}^{2}=0 \%$, $P_{\text {Heterogeneity }}=0.630 ;$ Arg399Gln: AA vs. GG, $\left.\mathrm{I}^{2}=0 \%, P_{\text {Heterogeneity }}=0.780\right)$, and allele (Arg399Gln: AA vs. GG, $\mathrm{I}^{2}=24 \%, P_{\text {Heterogeneity }}=0.250$ ) genetic models.

For subgroup analyses based on ethnicity, we found evidence of association between the Arg194Trp polymorphism and CAD susceptibility in Asian populations under recessive and homozygous genetic models, and support for an association for the Arg399Gln polymorphism under recessive, homozygous, and allele models of inheritance. Subgroup analyses stratified by control source found evidence of an association for both the Arg194Trp and Arg399Gln polymorphisms with increased likelihood of CAD in hospital-based studies under recessive and homozygous genetic models and a significant association between the Arg399Gln polymorphism and CAD susceptibility in population-based studies under recessive genetic model. In addition, we found in subgroup analyses stratified by sample size that findings related to the Arg194Trp polymorphism from studies with large sample sizes were similar to those found using pooled eligible studies (shown in Table 2).

\section{Sensitivity analysis}

The influence of each study on pooled ORs and $95 \%$ CIs were evaluated by excluding each study one at a time. As shown in Fig 4, we found no individual study affected the pooled OR.

\section{Publication bias}

Funnel plots (Fig 5) and Egger's linear regression test found no evidence of publication bias for either of the XRCC1 polymorphisms in Arg194Trp (TT + CT vs. CC, $P_{\text {Egger's }}=0.173$ ) or Arg399Gln (GA + AA vs. GG, $\left.P_{\text {Egger's }}=0.549\right)$.

\section{Discussion}

We analyzed ten studies in our meta-analysis and found evidence for associations between the Arg194Trp and Arg399Gln polymorphisms at the XRCC1 gene and an increased likelihood of CAD. Furthermore, subgroup analyses based on ethnicity found that carriers of the Arg194Trp T allele or the Arg399Gln A allele were at increased susceptibility to CAD in Asian populations under several genetic models. However, we found no evidence of an association in non-Asian populations, a finding that may indicate differences in genetic diversity between different 
A

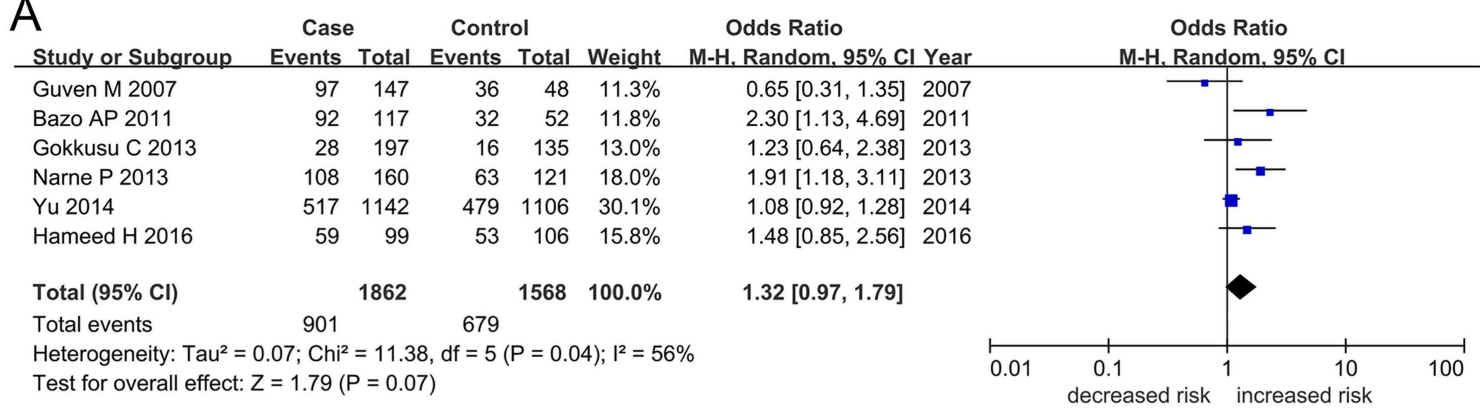

B

Case Control

Odds Ratio

Odds Ratio

Study or Subgroup Events Total Events Total Weight M-H, Fixed, 95\% Cl Yea

$\begin{array}{llllllll}\text { Guven M 2007 } & 21 & 147 & 3 & 48 & 4.1 \% & 2.50[0.71,8.78] 2007 \\ \text { Bazo AP 2011 } & 16 & 117 & 4 & 52 & 5.0 \% & 1.90[0.60,5.99] & 2011\end{array}$ Bazo AP 2011

Narne P 2013

Gokkusu C 2013

$16 \quad 117$

Yu 2014

$\begin{array}{lllll}24 & 160 & 14 & 121 & 14.3 \%\end{array}$

Hameed H 2016

$\begin{array}{rr}1 & 197 \\ 80 & 1142\end{array}$

$\begin{array}{rrr}14 & 121 & 14.3 \% \\ 0 & 135 & 0.6 \%\end{array}$

$1.35[0.67,2.73] 2013$

$2.07[0.08,51.16] 2013$

$1.31[0.93,1.86] 2014$

$1.65[0.88,3.08] 2016$

Total $(95 \% \mathrm{Cl})$

Total events

$31 \quad 99$

$1.65[0.88,3.08]$

Heterogeneity: $\mathrm{Chi}^{2}=1.50, \mathrm{df}=5(\mathrm{P}=0.91) ; \mathrm{I}^{2}=0 \%$

Test for overall effect: $Z=2.80(P=0.005)$

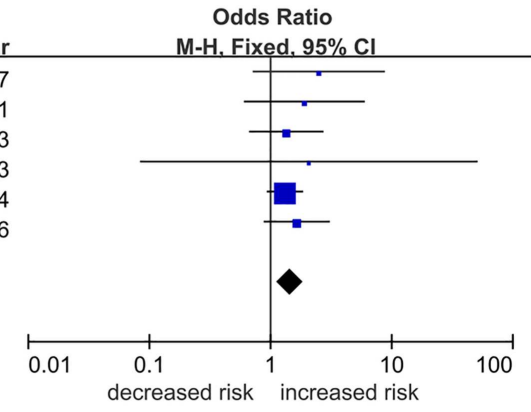

C

Case Control

Odds Ratio

Study or Subgroup Events Total Events Total Weight M-H, Random, 95\% Cl Year Guven M 2007

$\begin{array}{lllll}76 & 126 & 33 & 45 & 11.9 \%\end{array}$

Bazo AP 2011

Narne P 2013

Gokkusu C 2013

$\begin{array}{ll}76 & 101\end{array}$

$\begin{array}{lll}33 & 45 & 11.9 \% \\ 28 & 48 & 12.3 \%\end{array}$

$\begin{array}{lllll}84 & 136 & 49 & 107 & 18.0 \%\end{array}$

Yu 2014

$\begin{array}{lllll}27 & 196 & 16 & 135 & 13.9 \%\end{array}$

Hameed H 2016

$437 \quad 1062$

$\begin{array}{lll}419 & 1046 \quad 30.0 \%\end{array}$

Total $(95 \% \mathrm{Cl})$

Total events

$28 \quad 68$

$30 \quad 83 \quad 13.9 \%$

$0.55[0.26,1.17] 2007$

$2.17[1.05,4.51] 2011$

$1.91[1.14,3.20] 2013$

$1.19[0.61,2.30] 2013$

$1.05[0.88,1.24] 2014$

$1.24[0.64,2.39] 2016$

Heterogeneity: $\mathrm{Tau}^{2}=0.08 ; \mathrm{Chi}^{2}=11.42, \mathrm{df}=5(\mathrm{P}=0.04) ; \mathrm{I}^{2}=56 \%$

Test for overall effect: $Z=1.26(P=0.21)$

$1.23[0.89,1.70]$

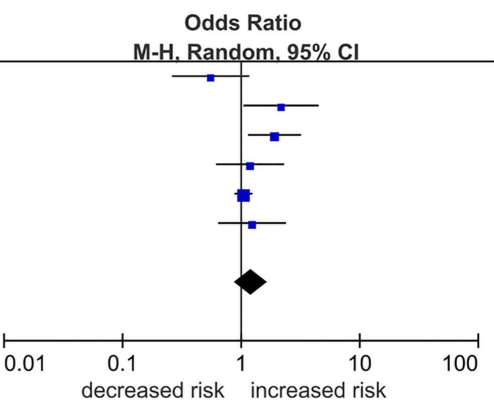

\begin{tabular}{|c|c|c|c|c|c|}
\hline Study or Subgroup & $\begin{array}{l}\text { Case } \\
\text { Events }\end{array}$ & Total & $\begin{array}{l}\text { Contrc } \\
\text { Events }\end{array}$ & & Weight \\
\hline Guven M 2007 & 21 & 71 & 3 & 15 & $4.2 \%$ \\
\hline Bazo AP 2011 & 16 & 41 & 4 & 24 & $3.7 \%$ \\
\hline Narne P 2013 & 24 & 76 & 14 & 72 & $11.8 \%$ \\
\hline Gokkusu C 2013 & 1 & 170 & 0 & 119 & $0.7 \%$ \\
\hline Yu 2014 & 80 & 705 & 60 & 687 & $64.6 \%$ \\
\hline Hameed H 2016 & 31 & 71 & 23 & 76 & $15.0 \%$ \\
\hline Total $(95 \% \mathrm{Cl})$ & \multicolumn{3}{|c|}{1134} & 993 & $100.0 \%$ \\
\hline Total events & 173 & & 104 & & \\
\hline \multicolumn{6}{|c|}{ Heterogeneity: $\mathrm{Chi}^{2}=2.49, \mathrm{df}=5(\mathrm{P}=0.78) ; \mathrm{I}^{2}=0 \%$} \\
\hline
\end{tabular}

Odds Ratio

M-H, Fixed, 95\% Cl Year $1.68[0.43,6.57] 2007$ $3.20[0.92,11.10] 2011$ $1.91[0.90,4.08] 2013$ $2.12[0.09,52.36] 2013$ $1.34[0.94,1.90] 2014$ $1.79[0.91,3.52] 2016$

$1.56[1.19,2.05]$

Test for overall effect: $Z=3.19(P=0.001)$

E

Case Control

\begin{tabular}{lrrrrrr} 
Study or Subgroup & Events & Total & Events & Total & Weight & M-H. Fixed, 95 \\
\hline Guven M 2007 & 118 & 294 & 39 & 96 & $6.1 \%$ & $0.98[0.61,1.57$
\end{tabular}

Guven M 2007

H. Fixed. $95 \% \mathrm{Cl}$ Yea

Bazo AP 2011

$108 \quad 234$

$\begin{array}{lll}36 & 104 & 4.7 \%\end{array}$

Narne P 2013

$132 \quad 320$

\begin{tabular}{lll}
$77 \quad 242 \quad 8.9 \%$ \\
\hline
\end{tabular}

Gokkusu C 2013

$29 \quad 394$

$\begin{array}{ll}16 & 270 \quad 3.1 \%\end{array}$

Yu 2014

$\begin{array}{rr}597 & 2284 \\ 90 & 198\end{array}$

$\begin{array}{lll}539 & 2212 \quad 70.3 \%\end{array}$

Hameed H 2016

$90 \quad 198$

$76 \quad 212 \quad 7.0 \%$

2007

$1.62[1.00,2.61] 2011$

$1.50[1.06,2.14] 2013$

$1.26[0.67,2.37] 2013$

$1.10[0.96,1.26] 2014$

$1.49[1.00,2.22] 2016$

Total $(95 \% \mathrm{Cl})$

1074

3724

Total events

Heterogeneity: $\mathrm{Chi}^{2}=6.60, \mathrm{df}=5(\mathrm{P}=0.25) ; \mathrm{I}^{2}=24 \%$

Test for overall effect: $Z=2.99(P=0.003)$

$1.18[1.06,1.32]$

$3136 \quad 100.0 \%$

(n)

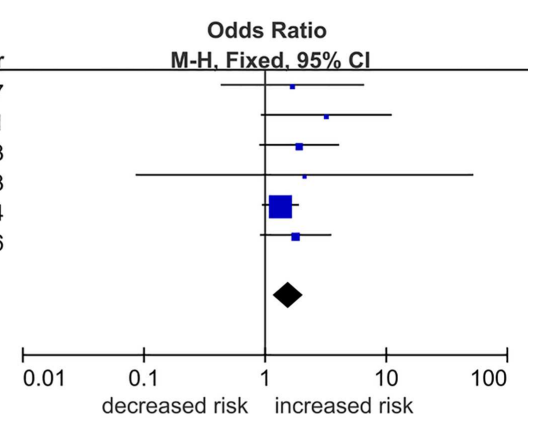

Odds Ratio M-H. Fixed, $95 \% \mathrm{Cl}$

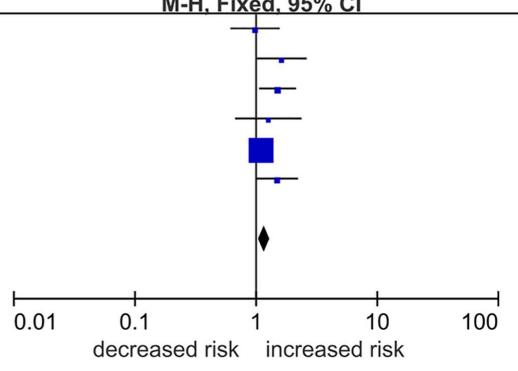


Fig 3. Forest plots for $X R C C 1$ Arg399GIn polymorphism and coronary artery disease susceptibility in the genetic models: (A) Dominant (GA + AA vs. GG), (B) Recessive (AA vs. GA + GG), (C) Heterozygous (GA vs. GG), (D) Homozygous (AA vs. GG), and (E) Allele (A allele vs. G allele). Abbreviations: XRCC1, X-ray repair cross complementing protein 1; $\mathrm{Cl}$, confidence interval.

doi:10.1371/journal.pone.0166961.g003

ethnic groups. Stratification based on control source found that both the Arg194Trp and Arg399Gln polymorphisms increased the likelihood of CAD in hospital-based studies under recessive and homozygous genetic models, and a significant association was observed between the Arg399Gln polymorphism and CAD susceptibility in population-based studies under recessive genetic model. Possible explanations for these discrepant findings may be the limitation of the small sample sizes, different risk factors among the hospital- and population-based controls, and the possibility of selection bias. In addition, it is worth noting that the hospitalbased controls are more 'case-like' than the population-based controls for many variables, which may underestimate the effects and can't represent the general population. NOS was applied for quality assessment and all the studies included were of moderate or high quality, which improved the reliability of the conclusion. Otherwise, studies of low quality might have increased the heterogeneity and produced misleading results. Furthermore, we performed sensitivity analysis and confirmed the robustness of our findings.

Although atherosclerosis is a complex process and its molecular mechanisms are not fully understood, research findings support the hypothesis that DNA damage caused by the production of excessive ROS has an important role in atherogenesis [26, 27], and that such ROSinduced DNA damage is coordinated to some extent by the XRCC1 pathway. Furthermore, polymorphisms in the $X R C C 1$ gene affect DNA repair efficiency and therefore, may influence individual susceptibility to both atherosclerosis and CAD. Consequently, our findings on the joint effects of these polymorphisms in the DNA repair gene XRCC1, on CAD susceptibility supports this rationale and is biologically plausible.

Ahmadi et al. [28] reported increased expression of the XRCC1 gene in patients with CAD when compared with normal people, and Martinet et al. [29] found a greater number of DNA strand breaks and an overexpression of the XRCC1 gene in atherosclerotic plaques. Such upregulation may be in response to elevated DNA damage because of atherosclerotic progression. These findings are consistent with the results of our meta-analysis that found XRCC1 gene polymorphisms may be susceptibility factors and outcome predictors to CAD. However, these findings are in contrast to those of Gokkusu et al. [18] and Guven et al. [23] that did not find evidence of a direct link between XRCC1 gene polymorphisms and CAD susceptibility. Possible explanations accounting for these inconsistencies between results may be because of genetic heterogeneity across different ethnicities, different baseline characteristics of study subjects, different sample sizes, or cultural differences in external factors such as nutritional status, diet, or daily exercise. On the other hand, the effect of a single polymorphism in the $X R C C 1$ gene on CAD susceptibility may be very small and is vulnerable to various factors. In recent decades, the dynamic equilibrium between DNA damage and repair in atherosclerosis has been highlighted. However, it remains to be determined whether reducing the rate of DNA damage and improving the capacity of DNA repair, such as overexpression of XRCC1 protein or gene replacement therapy in CAD patients with DNA repair deficiencies, can delay atherosclerotic progression, or open new avenues for therapeutic intervention [30, 31].

Previous meta-analyses have evaluated genetic polymorphisms in the XRCC1 gene and likelihood for different cancers including thyroid cancer [32], breast cancer [33], glioma [34], and leukemia [35], but to our knowledge, we have performed the first meta-analysis to examine the role of the XRCC1 gene in CAD susceptibility. Although our study provides a better understanding of genetic variations in $X R C C 1$ and susceptibility to $\mathrm{CAD}$, it has limitations. First, the 



Fig 4. Sensitivity analysis of the correlation between XRCC1 gene polymorphisms and susceptibility to coronary artery disease. (A) Sensitivity analysis for the Arg194Trp polymorphism. (B) Sensitivity analysis for the Arg399Gln polymorphism. Abbreviations: XRCC1, X-ray repair cross complementing protein 1; $\mathrm{Cl}$, confidence interval.

doi:10.1371/journal.pone.0166961.g004 


\section{A}



B



Fig 5. Funnel plots for studies investigating the effect of $X R C C 1$ polymorphisms on coronary artery disease susceptibility. (A) Funnel plot for publication bias in the Arg194Trp polymorphism. (B) Funnel plot for publication bias in the Arg399GIn polymorphism. Abbreviations: XRCC1, X-ray repair cross complementing protein 1; OR, odds ratio.

doi:10.1371/journal.pone.0166961.g005 
limited number of original studies that were examined and the unpublished data that were not available may affect the reliability of our conclusions and potentially limit further analyses. Second, because original individual data could not be extracted from each study and our results were based on unadjusted estimates, the introduction of heterogeneity in our study is unavoidable and may affect our results. Third, because of anticipated interactions between hereditary and environmental factors, genetic variation at a single locus will be insufficient to completely elucidate gene-disease associations. Finally, the publication language of our studies was limited to English and therefore, there is the potential for publication bias, although we found no evidence of such from funnel plots and Egger's test in our meta-analysis.

\section{Conclusions}

The current meta-analysis supports associations between the polymorphisms Arg194Trp and Arg399Gln, in the DNA repair gene XRCC1, and increased susceptibility to CAD, specifically in Asian populations. Although subgroup analyses to investigate potential sources of heterogeneity did not find evidence for bias, future large-scale well-designed studies to bolster the robustness of our findings and reliability of our conclusions are warranted.

\section{Supporting Information}

S1 File. meta-analysis-on-genetic-association-studies-form. (DOCX)

S2 File. PRISMA Checklist.

(DOC)

S3 File. The full details of databases searching terms.

(DOC)

S4 File. Full-text articles excluded with reasons.

(DOC)

S5 File. Eight excluded records.

(ZIP)

\section{Author Contributions}

Conceptualization: WQM NFL.

Data curation: WQM NFL XQH YZ.

Formal analysis: WQM NFL XQH YW.

Investigation: WQM NFL YZ.

Methodology: WQM NFL YZ.

Project administration: WQM NFL.

Resources: WQM NFL XQH XW YW YZ.

Software: YW XW.

Supervision: NFL.

Validation: WQM NFL XQH XW YW YZ.

Visualization: NFL. 
Writing - original draft: WQM.

Writing - review \& editing: WQM NFL.

\section{References}

1. Mozaffarian D, Benjamin EJ, Go AS, Arnett DK, Blaha MJ, Cushman M, et al. Heart disease and stroke statistics-2016 update: a report from the american heart association. Circulation. 2016; 133(4):e38360. doi: 10.1161/CIR.0000000000000350 PMID: 26673558

2. Husain $\mathrm{K}$, Hernandez $\mathrm{W}$, Ansari RA, Ferder L. Inflammation, oxidative stress and renin angiotensin system in atherosclerosis. World J Biol Chem. 2015; 6(3):209-17. doi: 10.4331/wjbc.v6.i3.209 PMID: 26322175

3. Palombo C, Kozakova M. Arterial stiffness, atherosclerosis and cardiovascular risk: Pathophysiologic mechanisms and emerging clinical indications. Vascul Pharmacol. 2016; 77:1-7. doi: 10.1016/j.vph. 2015.11.083 PMID: 26643779

4. Hooten NN, Ejiogu N, Zonderman AB, Evans MK. Association of oxidative DNA damage and C-reactive protein in women at risk for cardiovascular disease. Arterioscler Thromb Vasc Biol. 2012; 32(11):277684. doi: 10.1161/ATVBAHA.112.300276 PMID: 22982460

5. Cavallo D, Ursini CL, Setini A, Chianese C, Piegari P, Perniconi B, et al. Evaluation of oxidative damage and inhibition of DNA repair in an in vitro study of nickel exposure. Toxicol in Vitro. 2003; 17(5-6):6037. doi: 10.1016/s0887-2333(03)00138-3 PMID: 14599451

6. Yu E, Calvert PA, Mercer JR, Harrison J, Baker L, Figg NL, et al. Mitochondrial DNA damage can promote atherosclerosis independently of reactive oxygen species through effects on smooth muscle cells and monocytes and correlates with higher-risk plaques in humans. Circulation. 2013; 128(7):702-12. doi: 10.1161/CIRCULATIONAHA.113.002271 PMID: 23841983

7. Jackson SP, Bartek J. The DNA-damage response in human biology and disease. Nature. 2009; 461 (7267):1071-8. doi: 10.1038/nature08467 PMID: 19847258

8. Durante M, Bedford JS, Chen DJ, Conrad S, Cornforth MN, Natarajan AT, et al. From DNA damage to chromosome aberrations: Joining the break. Int J Hyg Environ Health. 2006; 209(6):503-11. doi: 10 1016/j.ijheh.2006.06.004 PMID: 16872898

9. Barnum KJ, O'Connell MJ. Molecular mechanisms involved in initiation of the DNA damage response. Mol Cell Oncol. 2015; 2(1):e970065. doi: 10.4161/23723548.2014.970065 PMID: 27308403

10. Tebbs RS, Flannery ML, Meneses JJ, Hartmann A, Tucker JD, Thompson LH, et al. Requirement for the xrcc1 DNA base excision repair gene during early mouse development. Dev Biol. 1999; 208 (2):513-29. doi: 10.1006/dbio.1999.9232 PMID: 10191063

11. Thompson LH, Brookman kW, Jones NJ, Allen SA, Carrano AV. Molecular cloning of the human XRCC1 gene, which corrects defective DNA strand break repair and sister chromatid exchange. Mol Cell Biol. 1990; 10(12):6160-71. PMID: 2247054

12. Whitehouse IJ, Taylor RM, Thistlethwaite A, Zhang H, Karimi-Busheri F, Lasko DD, et al. XRCC1 stimulates human polynucleotide kinase activity at damaged DNA termini and accelerates DNA single-strand break repair. Cell. 2001; 104(1):107-17. PMID: 11163244

13. Thompson LH, We MG. XRCC1 keeps DNA from getting stranded. Mutat Res. 2000; 459(1):1-18. PMID: 10677679

14. Shen MR JI, Mohrenweiser $\mathrm{H}$. Nonconservative amino acid substitution variants exist at polymorphic frequency in DNA repair genes in healthy humans. Cancer Res. 1998; 58(4):604-8. PMID: 9485007

15. Goode EL, Ulrich CM, Potter JD. Polymorphisms in DNA repair genes and associations with cancer risk. Cancer Epidemiol Biomarkers Prev. 2002; 11(12):1513-30. PMID: 12496039

16. Borghini $A$, Cervelli $T$, Galli A, Andreassi MG. DNA modifications in atherosclerosis: from the past to the future. Atherosclerosis. 2013; 230(2):202-9. doi: 10.1016/j.atherosclerosis.2013.07.038 PMID: 24075745

17. Andreassi MG. DNA damage, vascular senescence and atherosclerosis. J Mol Med. 2008; 86(9):103343. doi: 10.1007/s00109-008-0358-7 PMID: 18563380

18. Gokkusu C, Cakmakoglu B, Dasdemir S, Tulubas F, Elitok A, Tamer S, et al. Association between genetic variants of DNA repair genes and coronary artery disease. Genet Test Mol Biomarkers. 2013; 17(4):307-13. doi: 10.1089/gtmb.2012.0383 PMID: 23368530

19. Pahlavanneshan S, Ahmadi A, Boroumand M, Sadeghian S, Behmanesh M. Association of $-77 T>C$ and Arg194trp polymorphisms of XRCC1 with risk of coronary artery diseases in Iranian population. Iran J Basic Med Sci. 2016; 19(2):194-200. PMID: 27081465 
20. Yu X, Liu J, Zhu H, Xia Y, Gao L, Dong Y, et al. Synergistic association of DNA repair relevant gene polymorphisms with the risk of coronary artery disease in northeastern Han Chinese. Thromb Res. 2014; 133(2):229-34. doi: 10.1016/j.thromres.2013.11.017 PMID: 24315498

21. Narne $P$, Ponnaluri KC, Singh $S$, Siraj $M$, Ishaq $M$. Arg399gln polymorphism of $x$-ray repair cross-complementing group 1 gene is associated with angiographically documented coronary artery disease in south indian type 2 diabetic patients. Genet Test Mol Biomarkers. 2013; 17(3):236-41. doi: 10.1089/ gtmb.2012.0330 PMID: 23360319

22. Bazo AP, Jr DS, Salvadori RAF, Sodre LP, Silva GNd, Camargo EAd, et al. DNA repair gene polymorphism is associated with the genetic basis of atherosclerotic coronary artery disease. Cardiovasc Pathol. 2011; 20(1):e9-15. doi: 10.1016/j.carpath.2009.12.004 PMID: 20093049

23. Guven M, Guven GS, Oz E, Ozaydin A, Batar B, Ulutin T, et al. DNA repair gene XRCC1 and XPD polymorphisms and their association with coronary artery disease risks and micronucleus frequency. Heart Vessels. 2007; 22(6):355-60. doi: 10.1007/s00380-007-0986-9 PMID: 18043991

24. Stang A. Critical evaluation of the Newcastle-Ottawa scale for the assessment of the quality of nonrandomized studies in meta-analyses. Eur J Epidemiol. 2010; 25(9):603-5. doi: 10.1007/s10654-0109491-z PMID: 20652370

25. Hameed H, Faryal M, Aslam MA, Akbar A, Saad ABA, Pasha MB, et al. Association of polymorphisms (rs 1799782, rs25489 and rs25487) in XRCC1 and (rs 13181) XPD genes with acute coronary artery syndrome in subjects from multan, pakistan. Pak J Pharm Sci. 2016; 29(3):869-76. PMID: 27166553

26. Hirata Y, Yamamoto E, Tokitsu T, Kusaka H, Fujisue K, Kurokawa H, et al. Reactive oxygen metabolites are closely associated with the diagnosis and prognosis of coronary artery disease. J Am Heart Assoc. 2015; 4(2). doi: 10.1161/JAHA.114.001451 PMID: 25630910

27. Harrison D, Griendling KK, Landmesser U, Hornig B, Drexler H. Role of oxidative stress in atherosclerosis. Am J Cardiol. 2003; 91(3):7-11. doi: 10.1016/s0002-9149(02)03144-2 PMID: 12645638

28. Ahmadi A, Behmanesh M, Boroumand MA, Tavallaei M. Up-regulation of MSH2, XRCC1 and ATM genes in patients with type 2 diabetes and coronary artery disease. Diabetes Res Clin Pract. 2015; 109 (3):500-6. doi: 10.1016/j.diabres.2015.05.049 PMID: 26088318

29. Martinet W, Knaapen MWM, De Meyer GRY, Herman AG, Kockx MM. Elevated levels of oxidative DNA damage and DNA repair enzymes in human atherosclerotic plaques. Circulation. 2002; 106(8):927-32. doi: 10.1161/01.cir.0000026393.47805.21 PMID: 12186795

30. Cervelli T, Borghini A, Galli A, Andreassi MG. DNA damage and repair in atherosclerosis: current insights and future perspectives. Int J Mol Sci. 2012; 13(12):16929-44. doi: 10.3390/ijms131216929 PMID: 23443128

31. Shah NR, Mahmoudi M. The role of DNA damage and repair in atherosclerosis: A review. J Mol Cell Cardiol. 2015; 86:147-57. doi: 10.1016/j.yjmcc.2015.07.005 PMID: 26211712

32. Wu FF, He XF, Shen HW, Qin GJ. Association between the XRCC1 polymorphisms and thyroid cancer risk: a meta-analysis from case-control studies. PloS one. 2014; 9(9):e87764. doi: 10.1371/journal. pone.0087764 PMID: 25211472

33. Bu T, Liu L, Sun Y, Zhao L, Peng Y, Zhou S, et al. XRCC1 Arg399Gln polymorphism confers risk of breast cancer in American population: a meta-analysis of 10846 cases and 11723 controls. PloS one. 2014; 9(1):e86086. doi: 10.1371/journal.pone.0086086 PMID: 24489692

34. Jacobs DI, Bracken MB. Association between XRCC1 polymorphism $399 \mathrm{G}->\mathrm{A}$ and glioma among Caucasians: a systematic review and meta-analysis. BMC Med Genet. 2012; 13:97. doi: 10.1186/14712350-13-97 PMID: 23101479

35. Zhang H, Liu H, Jiang G. Genetic polymorphisms of XRCC1 and leukemia risk: a meta-analysis of 19 case-control studies. PloS one. 2013; 8(11):e80687. doi: 10.1371/journal.pone.0080687 PMID: 24363792 